\title{
Numerical Prediction of Tonal Noise Generation in an Inlet Vaned Low-Speed Axial Fan Using a Hybrid Aeroacoustic Approach
}

\author{
Argüelles Díaz, K.M.; Fernández Oro, J.M.; \\ Blanco Marigorta, E.; Santolaria Morros, C. \\ Universidad de Oviedo, Área de Mecánica de Fluidos. \\ Campus de Viesques, 33271, Gijón (Asturias), Spain. \\ arguelleskatia@uniovi.es
}

\begin{abstract}
This work presents a numerical prediction of the tonal noise generation in a single stage, axial flow fan, using a hybrid approach that firstly calculates the noise sources (generation) using conventional CFD techniques, and then estimates the noise level in the blower far-field region (propagation) by means of an aeroacoustic analogy. As a starting point, an unsteady 3D full-annulus simulation of the internal flow is carried out, using a WMLES scheme for the turbulence closure to identify the acoustic sources. A well-tested commercial CFD package, FLUENT, was employed for that purpose, so a complete set of unsteady forces exerted over the blades was calculated. Following, a generalization of the Lighthill's aeroacoustic analogy, the so-called Ffowcs Williams and Hawkings aeroacoustic analogy (FFWH), was numerically implemented using a $C++$ algorithm to resolve an integral formulation of the free-field FFWH wave equation, where CFD data is included in the source terms. At this point, both temporal and frequential domains were considered in the methodology, because the comparison of their results allowed the segregation of the specific contribution of the blade's thickness noise in the total noise spectra. The major contribution was expected to be found in the estimation of the tonal noise levels, directly linked to the intensity of the stator-rotor interaction phenomena. Different axial gaps between the fixed and rotating blade rows of the fan were considered and clear conclusions were obtained relating the interrow spacing and the tonal noise magnitude. Additionally, intensive experimental measurements in the noise propagation area of the fan were conducted, in order to validate the numerical study. A reasonable agreement was found in the tonal noise spectra, though important discrepancies appeared due to the attenuation produced by the fan casing, not considered in the numerical model. However, the numerical study provided valuable information about the particular influence of the tonal noise sources, estimating accordingly overall experimental trends, and showing the potentiality of numerical tools to deal with noise control for designers and researchers.
\end{abstract}

\section{INTRODUCTION}

Nowadays, the reduction of the noise levels emitted by aircraft engines is a key parameter in the development of new designs for manufacturers and researchers. During the last decades, the optimization of propulsion systems in airplane engines led to important reductions of the jet noise, while other acoustic sources, like turbofan noise, were still an issue to deal with. However, progressive noise restrictions, especially in international airports, have motivated the development of new methods to identify sources of noise and its mechanisms of propagation. Different experimental and numerical methodologies have arisen recently to provide further knowledge of the noise sources in turbomachinery, most of them considering high-speed turbomachinery used in the aeronautic industry [1]. Up to now, just a few works have analyzed in detail noise patterns in blowers and lowspeed axial fans ([2], [3], [4]).
Two different strategies can be considered to characterize both generation and propagation phenomena of the aerodynamic noise inside a turbomachine: a direct approach or a hybrid approach. The direct approach allows a complete description of all the flow field phenomena through a direct numerical simulation (DNS) of the complete unsteady Navier-Stokes equations for the compressible regime. Nevertheless, this method, which is basically used in problems of Computational Aeroacoustics (CAA), requires extremely high computational resources if an accurate precision is desired for the resolution (for both calculation efforts and time necessary to achieve a complete solution of the flow and acoustic fields) [5]. This is due to the disparity between the temporal and the spatial scales that exist for both flow field phenomena (i.e. turbulence) and acoustic characteristics (noise propagation). Therefore, this kind of methodologies has been employed only with simple, two-dimensional geometries. Unfortunately, the 
complexity of the three-dimensional phenomena involved in any axial turbomachine is far beyond the scope of this approach.

The hybrid approach is presented as an alternative to the direct approach and takes into account the difference of the temporal and spatial scales between the flow field and the acoustic field. This consideration allows a segregation of the complete problem of both generation and propagation of the aerodynamic noise into two separate problems. First, the acoustic sources and the flow field must be determined, and second, the calculation of the acoustic field is completed as a direct consequence of those sources [6].

There are several hybrid approaches, but the most successful one, in terms of accuracy in capturing acoustic characteristics of turbomachinery, is the Lighthill's aeroacoustic analogy. This analogy divides the interest domain in two sub-domains: the source region and the propagation region [7], [8]; and it also transforms the Navier-Stokes equations into a wave equation which includes source terms as real acoustic sources. In the same fashion, the Ffowcs Williams and Hawkings' aeroacoustic analogy (FFWH) is just an extension of the Lighthill's analogy which includes the possibility of introducing moving bodies inside the source region [9]. This way, using FFWH analogy, the flow field patterns can be obtained using conventional CFD modelling techniques and the acoustic sources can be identified in the procedure. After that, under free field conditions, the noise propagation into the far field can be resolved using a numeric resolution of an integral formulation of the FFWH wave equation.

In the regular performance of an axial fan, mechanisms of noise generation at discrete frequencies (tonal noise) superimposed to broadband noise contribute to the sound spectrum. Tonal noise is characterized by the presence of higher peaks at the BPF and its harmonics, while broadband noise, associated with the acoustic energy generated by nondeterministic scales, is distributed along the whole range of frequencies. Additionally, noise phenomena can be classified according to the characteristics of its propagation, or directivity, identifying monopolar sources (associated to thickness noise), dipolar (caused by the forces exerted by the flow over the blades) and quadrupolar (due to the flow turbulent phenomena) [10].

The present work is focused on the generation and propagation of discrete noise in a low-speed axial fan. Particularly, the most important mechanism of generation of tonal noise in turbomachinery is treated in detail: the unsteady aerodynamic interaction between fixed and moving blade rows (stator-rotor interaction). This feature is responsible for the existence of dipolar sources, known as "discrete loading noise", and monopolar sources, referred as "thickness noise", primary contributors that will be analyzed as a function of the interrow gap. For that purpose, a control surface matching with the rotor blades surfaces has been defined to model both dipolar and monopolar sources. To obtain the acoustic sources, a commercial CFD code (FLUENT) has been employed, developing a real 3D unsteady simulation which includes a sliding mesh technique.

The numeric characterization of the acoustic propagation region placed at the far field -the zone of real interest- has been implemented using a $\mathrm{C}++$ algorithm, through an integral formulation of the freefield FFWH wave equation. The source terms of the wave equation are obtained from afore mentioned CFD data. The integral formulation of the FFWH equation has been resolved in both frequential [11] and temporal [12] domains.

Finally, the validation of the present methodology has been tested with a set of experiments to check the accuracy of the numerical results in both generation and propagation sub-domains. In the source region, dual hot wire anemometry has been employed intensively to validate the CFD modelling [13]. Significant efforts were devoted to obtain a reliable model because it is well-known that much of the precision in the results of the propagation region are a consequence of a correct modelization of the acoustic sources [14]. In the propagation zone, experimental measurements of the noise level have been completed using capacitive microphones with a spectrum analyzer to obtain the contribution of the tonal sources. Also, the comparison of the numerical and experimental results has allowed the quantification of the importance of the casing attenuation or the ground effect.

\section{EXPERIMENTAL FACILITY}

The generation and propagation of tonal noise due to stator-rotor interaction has been studied in an inlet vaned axial flow fan. The axial blower under consideration is composed of a single stage 9-blade rotor (NACA 65 profile) placed downstream of a 13 IGVs stator (circular profile BRITISH C1), which straightens the flow towards the rotor. The rotational speed of the machine is $2400 \mathrm{rpm}$, so the blade passing frequency $(\mathrm{BPF})$ is $360 \mathrm{~Hz}$. The nominal flow rate was fixed by design at $18 \mathrm{~m}^{3} / \mathrm{s}$, for a total-to-static pressure rise of $1200 \mathrm{~Pa}$. The hub and tip diameters of the blower are $380 \mathrm{~mm}$ and $820 \mathrm{~mm}$, respectively. Figure 1 shows a sketch of this axial configuration, with basic parameters of the fan stage.

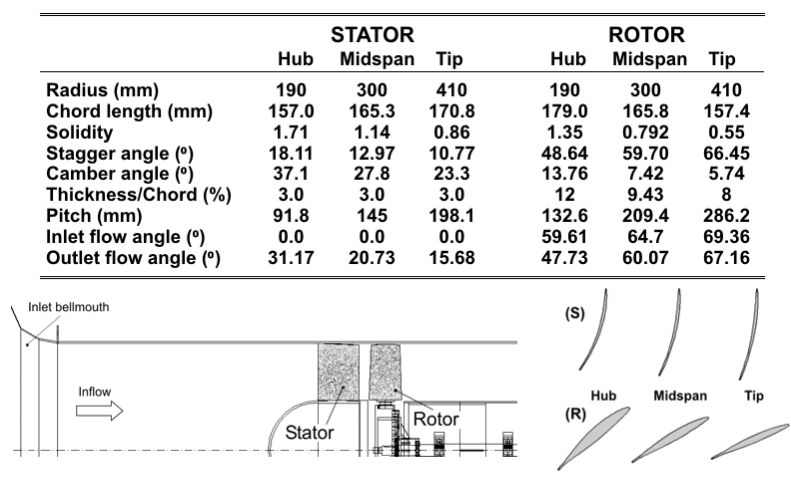

Figure 1. Sketch and basic parameters of the axial fan stage. 
Additionally, the fan has been constructed with the possibility of changing the axial gap between the rows. The stator-rotor separation is modifiable through a set of annular rings that can be removed or added in the fan shroud to decrease or increase the gap. Two axial gap configurations were tested in the present study: a larger nominal gap of $50 \%$ of the blade chord, and a lower gap of a $37 \%$ of the blade chord.

Hot-wire measurements were conducted in a test facility in the lab, under controlled environment conditions [15]. Conversely, the estimation of noise levels using capacitive microphones was carried out in nearly free-field conditions, outside the lab. This validation has been completed using $1 / 2$ " Brüel\&Kjaer capacitive microphones and a spectrum analyzer (B\&K, 2133). Figure 2 shows a photograph of the experimental set-up and the definition of the measuring locations in the propagation region. Three different radial positions, at 5, 7 and 9 meters from the axial fan were considered to analyze the progressive attenuation of the noise level. The uncertainty of the experimental measurements is $\pm 2 \mathrm{~dB}$, for a confidence interval of $95 \%$. Notice that this uncertainty is not introduced in the figures in order to make the results' visualization clearer. Also, the ground effect on the noise measurements has been considered as an overestimation in the noise levels of approximately 3 $\mathrm{dB}$ according to [16].
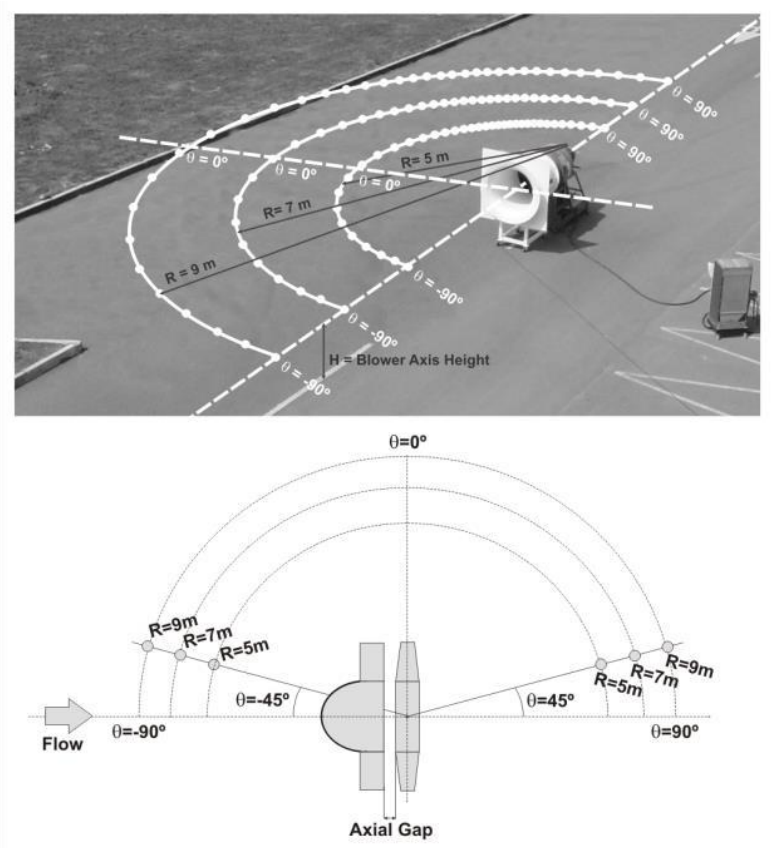

Figure 2. Experimental set-up and measurement points.

\section{NUMERICAL METHODOLOGY}

The numerical prediction of the tonal noise generation in a single stage, axial flow fan, using a hybrid approach that firstly calculates the noise sources (generation) using conventional CFD techniques, and then estimates the noise level in the blower far-field region (propagation) by means of an aeroacoustic analogy. Figure 3 presents a flow chart describing this methodology. Following, details of the CFD technique and the aeroacoustic analogy are provided.

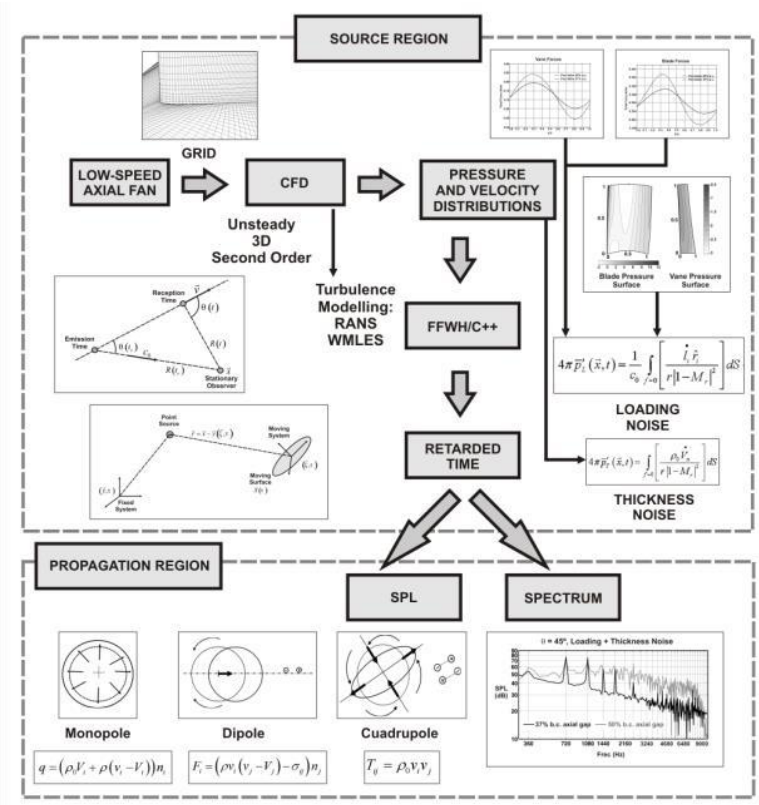

Figure 3. Flow chart of the computational procedure.

\section{CFD technique}

Geometry, mesh and solver. The commercial CFD software FLUENT v6.1 was used to solve the NavierStokes set of equations. The code, unsteady, viscous and three-dimensional, introduces a cell-centered volume finite method with an explicit, second-order accurate scheme for the temporal discretization. A SIMPLE algorithm is chosen for the pressure and velocity coupling, and central differencing discretizations have been used for convection and diffusion terms. The continuity and momentum equations for a three-dimensional flow are:

$$
\frac{\partial \vec{W}}{\partial t}+\nabla \vec{F}=\vec{H}
$$

where $\vec{W}$ is the vector of conservation variables, $\vec{F}$ is the flow vector and $\vec{H}$ is the source terms vector:

$$
\vec{W}=\left[\begin{array}{c}
\rho \\
\rho v_{1} \\
\rho v_{2} \\
\rho v_{3}
\end{array}\right] \quad \vec{F}=\left[\begin{array}{c}
\rho v_{j} \\
\rho v_{1} v_{j}+p \vec{i}-\tau_{1 j} \\
\rho v_{2} v_{j}+p \vec{j}-\tau_{2 j} \\
\rho v_{3} v_{j}+p \vec{k}-\tau_{3 j} \\
j=1,2,3
\end{array}\right] \quad \vec{H}=\left[\begin{array}{c}
0 \\
S_{M_{1}} \\
S_{M_{2}} \\
S_{M_{3}}
\end{array}\right]
$$

In previous equations, $p$ is the static pressure, $\rho$ is the flow density, $t$ is the time, $v_{j}$ are the velocity components, $S_{M i}$ are the momentum source terms and $\tau_{i j}$ is the viscous stress tensor. 
A compatible pre-processor, GAMBIT v2.0, was used to develop both geometries and meshes. No simplifications for the relative number of blades (9) and vanes (13) were adopted, so a full 3D annulus grid of the stage was implemented with 22 total passages and 2 million cells for the whole domain. The final discretization was fixed according to a grid sensibility analysis, performed during the preliminary tests (figure

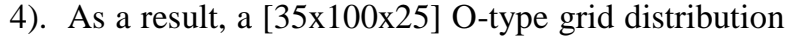
was introduced for every passage, increasing the grid resolution near the surfaces. The 25-node radial distribution could not allow a tip clearance gridding (rotor casing rotates along with the blades domain), but it was optimized to guarantee a better flow description in the hub and tip sections. Figure 3 also shows that cells were extruded spanwise from hub to tip, even in the rotor passages, where blade twisting provokes high skewed elements.

Using a "sliding-mesh" technique, the unsteady simulation provides a temporal description of the flow variables throughout the stage. Thereby, the rotor zone is forced to rotate around the axis at $2400 \mathrm{rpm}$. In order to obtain a good resolution for the velocity fields, the time step was fixed at $5.3418 \times 10^{-5} \mathrm{~s}$.

Turbulence closure. WMLES vs RANS. The incompressible Navier-Stokes equations are always closed using a turbulence modeling in any numerical simulation. As a commercial code, FLUENT provides a wide range of turbulent models, from the classical Reynolds-averaged schemes (RANS) to large eddy simulation (LES) techniques. In this study, both RANS modeling and LES techniques were tested to find the most accurate scheme for the simulation.

An unsteady RANS (URANS) model was first introduced. This scheme models all turbulence scales, solving ensemble-averaged Navier-Stokes equations. RANS modeling offers different possibilities (SpalartAllmaras, k- $\varepsilon, \mathrm{k}-\omega, \mathrm{RSM}$ ) according to increasing complexity. In this case, a Reynolds Stress Model (RSM) has been chosen because of its suitable predictions for rotating flow passages. Effects of curvature, swirl and rotation are directly accounted for in the transport equation, and the turbulence is considered anisotropic.

Near-wall modeling has been introduced with a standard logarithmic wall function, so wall-adjacent cells are not placed in the buffer layer $(y+\sim 30)$. Outer region and log-law layer are properly resolved by the RSM scheme near the walls. Considering a Reynolds number based on tip characteristics $\left(\operatorname{Re}_{\mathrm{Ch}}=12 \times 10^{5}\right)$, the first grid point is placed at $0.2 \mathrm{~mm}$ from the blade surfaces, with a growth factor ratio of 1.3. As a result, a $y+$ value of 60 wall units was fixed at both suction and pressure side of the blades.

Using URANS results as initial conditions, a LES technique was attempted to resolve the larger eddies. In this case, the governing equations are grid-filtered so subgrid-scale stress terms are introduced into the model. The subgrid-scale stresses are unknown and require modeling. Thus, a Smagorinsky-Lilly model has been used, with a default value of $\mathrm{C}_{S}=0.1$. The requirement for a well-resolved LES in the near-wall region expressed in wall units is approximately $\Delta \mathrm{x}+\sim$ 100 (streamwise) and $\Delta \mathrm{z}^{+} \sim 20$ (spanwise). Also, the near-wall grid spacing should be about one wall unit in the wall-normal direction, $\Delta y+\sim 1$ ([17]). These grid requirements are not affordable for actual industrial computational capacities in the case of a full annulus $3 \mathrm{D}$ domain. It can be said that wall-bounded flows at high Reynolds numbers need computational resources prohibitively large for pure LES algorithms.

To avoid the extreme requirement of near-wall grid resolution, the LES scheme is used including near-wall modeling below the inner part of the logarithmic region (between 30 to 60 wall units away from the wall). Then, the near-wall region is sufficiently resolved and hence the near-wall model is automatically applied by FLUENT in the LES simulations, resulting in a wallmodeled large eddy simulation (WMLES). This approach allows the first grid point to be located in the log layer, but the vortical structures in both viscous and buffer regions are not resolved, and therefore coarse meshes can be used in all the directions $(\Delta \mathrm{x}+\sim 100$ $\left.600, \Delta z^{+} \sim 100-300\right)$. This means that the interaction between the modeled, near-wall region, and the resolved, outer region, is weak, assuming that only the Reynolds shear stresses are significant. Thus, the stress at the wall is computed in terms of the velocity at the first inner point, and the dynamics of the wall layer is considered to be more universal [18].

Time step size is another restrictive parameter for LES techniques. Generally, it should be small enough to resolve the time-scale of the smallest resolved eddies. According to the mesh density, the order of magnitude of these eddies is that of the wakes' shear layer at the blade's trailing edge. Considering the through-flow velocity as a representative value, at least one time step in the range of $10^{-5} \mathrm{~s}$ must be fixed to capture eddies shedding.

Obviously, numerical simulations with LES algorithms require finer meshes and lower time steps if complete vortex shedding needs to be described. Otherwise, just larger eddies are captured and resolved. However, an initial approach to LES techniques has been explored in this study, resulting in a description of the unsteady stator-rotor interaction that follows previous experimental data. In this case, WMLES allows the calculation to reflect the instability of the shear layer and the development of the coherent structures in the wake. In addition, more accurate prediction of the unsteady forces and of the sound emission can be obtained when compared to steady or unsteady pure RANS methods [18].

BC's and overall convergence. Finally, both pressure conditions at the inlet (total-pressure) and the outlet (static-pressure) of the domain were established to set flow boundary conditions as neutral as possible. This enhances the quality of the numerical solutions, approaching it to the real physical process. For WMLES computations, the stochastic (non-modeled) 
components at the flow boundaries are accounted for by superposing random perturbations, based on a Gaussian random function. Unfortunately, convergence history is badly-affected by this election, increasing the real time required for the model to converge (residual criterion in continuity of $10^{-6}$ ), especially at points far from design conditions. However, the numerical performance curve of the $3 \mathrm{D}$ model has presented overall good results in comparison to the performance curves of the real machine (taking into account that tip clearance has not been considered), when the working point is close to the best efficiency point (not shown here).

For every working point, $350 \mathrm{~h}$ of CPU time were necessary to obtain a periodic stable response of the model in the WMLES computations in an 8 PC cluster, $2.6 \mathrm{GHz}$, parallelized with a Gigabit connection set. Once the periodicity was achieved (generally after three or four rotor turns), a final running of the model was carried out to store the velocity and pressure maps during a complete blade passage (52 angular phases per rotor passage).
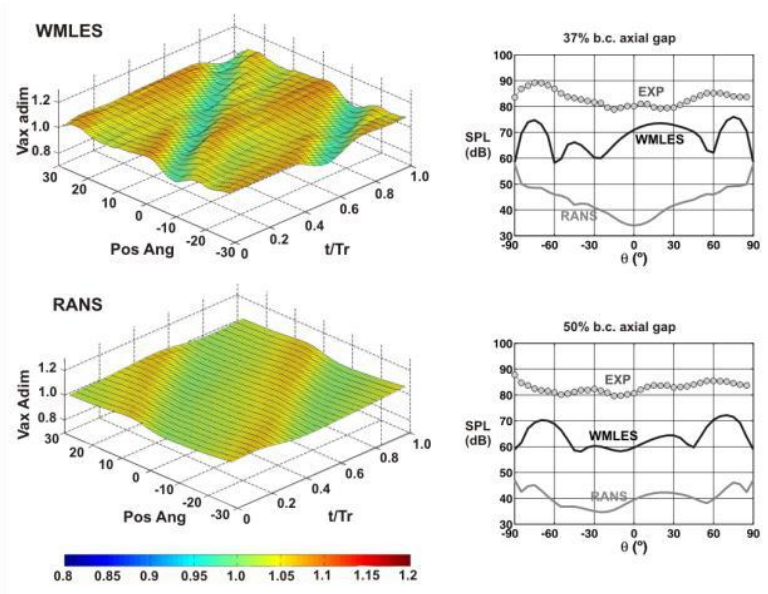

Figure 5. Turbulence modelling comparison.

Figure 5 is a composed picture showing the instantaneous velocity patterns downstream of the rotor for a complete blade passing period in the numerical model. Results for both WMLES and RANS modelling have been represented to illustrate the major accuracy provided by the WMLES technique. In this study, the URANS solution has demonstrated greater numerical diffusion (notice the excessive smoothness of the wake deficit in the RANS plot) and therefore poor predictions of the acoustic noise than WMLES computations. More details about the accuracy of the CFD modelling can be found in [19]. Figure 5 also shows the comparison of the total sound pressure level obtained with the two sets of unsteady pressure distributions over the blades (provided by both turbulence closures) after applying the aeroacoustic analogy (explained below). Recent investigations in CAA, pointing out that the LES scheme is capable of capturing more precisely the aeroacoustic scales than a typical RSM model, seems to be confirmed in the present work. In essence, the main difference between a classical turbulent model and the LES scheme is the way the vortical structures are modelled: classical RANS turbulence closure terms model the complete range of the vortical structures of the flow, whereas the LES scheme establishes a scale cut-off for a spatial filtering, modelling just those vortical structures whose characteristic scale is below the cut-off limit. The upper structures are resolved directly, so this characteristic enhances the resolution and also increases the complexity of the formulation. Thus, the LES scheme improves computational precision, because just the lowest scales are modelled, and it is accepted that it is easier to formulate a universal model for the lowest scales of turbulence, more isotropic and less dependent on boundary conditions of the numerical modelling. Because the acoustic scales have more affinity with the higher scales of turbulence, it is quite logical to expect better behaviour of the LES scheme when carrying out the numerical predictions of the sound field.

\section{FFWH analogy}

The formulation of FFWH aeroacoustic analogy has been employed to resolve the generation and the propagation of aerodynamic noise in this blower.

This analogy is an extension of the well-known Lighthill's analogy to take into account the effect of moving bodies in the source region. In this case, and opposite to the original Lighthill's formulation where just the flow is replaced by equivalent acoustic sources, both flow patterns and moving surfaces require such replacement.

For an accurate representation of the real medium, it is necessary to define an equivalent medium where all the rigid bodies are replaced by control volumes. It is assumed that those volumes contain the surrounding fluid at rest. In addition, the flow kinematics and the boundaries preserving the no-slip condition at the surfaces are imposed introducing a discontinuity in space. This is achieved with moving mass and momentum sources in the fluid dynamic equations.

This procedure gives an inhomogeneous wave equation for the acoustic pressure fluctuations $p^{\prime}$. When the control surface matches with a solid contour, the equation is formulated as follows:

$$
\begin{gathered}
\frac{1}{4 \pi c_{0}^{2}} \frac{\partial^{2}}{\partial t^{2}}\left(p^{\prime} H(f)\right)-\frac{1}{4 \pi} \frac{\partial^{2}}{\partial x_{i}^{2}}\left(p^{\prime} H(f)\right)= \\
=\frac{\partial^{2}}{\partial x_{i} \partial x_{j}}\left[T_{i j} H(f)\right]+\frac{\partial}{\partial t}\left[\rho_{0} V_{i} n_{i}|\nabla f| \delta(f)\right]+ \\
+\frac{\partial}{\partial x_{i}}\left[\sigma_{i j} n_{j}|\nabla f| \delta(f)\right] \\
i=1,2,3 \quad j=1,2,3
\end{gathered}
$$

where $f(\vec{x}, t)=0$ is the control surface, $H(f(\vec{x}, t))$ is the Heaviside function, $\rho$ is the medium density, 
$\rho_{0}$ is the non-perturbed medium density and $c_{0}$ is the speed of sound. Resides, $V_{i}$ and $n_{i}$ are the velocity of the control surface and its normal unit, $\delta(f)$ is the Dirac function and $\sigma_{i j}=-p^{\prime} \delta_{i j}+\tau_{i j} \quad$ and $T_{i j}=\rho v_{i} v_{j}-c_{0}^{2} \rho^{\prime} \delta_{i j}-\sigma_{i j}$ are the fluid and Lighthill's stress tensors respectively.

Accordingly to Lighthill's analogy, equation (3) cannot be applied directly to determine the acoustic field because there are acoustic variables in the source terms. Therefore, it is necessary to impose some hypothesis to transform this equation in a real wave equation to be solved using a standard technique based on Green functions.

The first source term represents a volume distribution that corresponds to the Lighthill's stress tensor with a quadrupolar character. Under the assumption of isentropic flow, with a high Reynolds number and low Mach number conditions, $\mathrm{T}_{\mathrm{ij}}$ is reduced to:

$$
T_{i j}=\rho_{0} v_{i} v_{j}
$$

The second source term represents a surface distribution fully determined by the kinematics of body forces. In this case, no additional simplifications are required. Typically known as thickness noise, it presents a monopolar directivity according to:

$$
q=\rho_{0} V_{i} n_{i}
$$

Finally, the third source term is also corresponding to a surface distribution, now coming from the interaction between the flow and the moving bodies. It represents the fluid-structure forces, but also includes the effect of those surfaces over the acoustic waves. These means that they cannot be fully determined if the acoustic field is still unknown. Fortunately, the fluctuating forces coming from the rotating blades are significantly higher than the acoustic perturbations, so these reflection mechanisms can be easily neglected because the noise generation (and not its diffraction) is the primary contributor. This source term is usually known as loading noise, with a dipolar characteristic and the following mathematical expression:

$$
F_{i}=-\sigma_{i j} n_{j}
$$

Applying directly the Green's function technique, as well as the Green function of the free field, an integrated formulation of the FFWH equation, known as Farassat 1A formulation, is obtained in the retarded time (i.e., the observer's time). This formulation is not considering the quadrupolar (or volumetric) source term -only representative for high-speed turbomachines- due to the subsonic condition of the present investigation. Therefore, the acoustic pressure fluctuations are calculated as follows:

$$
p^{\prime}(\vec{x}, t)=p_{T}^{\prime}(\vec{x}, t)+p_{L}^{\prime}(\vec{x}, t)
$$

where $p_{T}^{\prime}(\vec{x}, t)$ and $p_{L}^{\prime}(\vec{x}, t)$ correspond to the thickness and loading noise respectively. For an observer placed in the far field region, they are fyrther evaluated by:

$$
\begin{gathered}
4 \pi p_{T}^{\prime}(\vec{x}, t)=\int_{f=0}\left[\frac{\rho_{0} \dot{V}_{n}}{r\left|1-M_{r}\right|^{2}}\right] d S \\
4 \pi p_{L}^{\prime}(\vec{x}, t)=\frac{1}{c_{0}} \int_{f=0}\left[\frac{\dot{l}_{i} \hat{r}_{i}}{r\left|1-M_{r}\right|^{2}}\right] d S
\end{gathered}
$$

The dot over the variables in equations (8) and (9) represent the differencing operator respect to the source (or surface) time, while $n$ and $r$ are referring to the scalar product of the corresponding variable with the normal unit of the surface and the normal unit in the radiating direction respectively. In addition, $l_{i}$ is the local force exerted by the fluid over the blade surfaces, $S$ is the control surface, $M$ stands for the Mach number and $\vec{r}$ is the position vector for the direction of the radiating noise, while the brackets indicate that its evaluation must be done in the retarded time (or emission time) according to:

$$
g=\tau-t+r / c_{0}
$$

where $\tau$ is the source time and $t$ is the time of the observer. Precisely, the resolution of the equation for the retarded time (10) implies extremely high computational costs for any algorithm to calculate the noise propagation into the far field. Fortunately, this inconvenience can be avoided if the wave equation (3) is resolved in the frequency domain, instead of the temporal formulation.

In the frequency domain, just the influence of the discrete loading noise is considered as a result of the pressure fluctuations over the rotor blades over time (implemented as a loading spectrum). In this case, the integrated solution of the FFWH equation, when considering a stator-rotor interaction, is established as follows (see [11] for a complete review):

$$
\begin{aligned}
p^{\prime}(\vec{x}, t) & =\sum_{m=-\infty}^{\infty} \sum_{w=-\infty}^{\infty} \frac{i m B^{2} \Omega}{4 \pi R c_{0}} e^{-i m B \Omega t} e^{i m B \Omega R / c_{0}} e^{i(m B-w V)(\varphi-\pi / 2)} \times \\
& \int_{0}^{2 \pi} \int_{R_{H}}^{R_{T}} F_{w V} e^{-i(m B-w V) \varphi_{0}} \times J_{m B-w V}\left(\frac{m B \Omega R_{0}}{c_{0}} \sin \theta\right) \times \\
& \times\left[\cos \gamma \cos \theta-\frac{m B-w V}{m B} \frac{c_{0}}{\Omega R_{0}} \sin \gamma\right] R_{0} d R_{0} d \varphi_{0}
\end{aligned}
$$

where $m$ refers to the harmonics of the BPF, the $w$ index represents the load harmonics of the blade $F_{w V}, B$ is the number of rotor blades, $V$ is the number of stator 
vanes, $\Omega$ is the rotation speed of the fan and $(R, \theta, \varphi)$ are the spherical coordinates of the observer position in the far field. Also, $R_{0}$ is the spanwise position of every blade, $\varphi_{0}$ is the streamwise location along the blade chord, $\gamma$ is the inclination angle -relative to the rotation axis-, and $R_{H}$ and $R_{T}$ are the rotor hub and tip radii respectively. Notice that this complex equation introduces Bessel functions, $J$, of order $m B-w V$, inherently related to the number of blades and vanes following the Tyler and Sofrin rule.

\section{Propagation region}

The numerical characterization of the propagation region can be carried out in both temporal (equations [7]-[10]) and frequency domains (equation [11]).

In the frequency domain, just the influence of the discrete loading noise is considered; meanwhile the temporal domain considers the influence of both discrete loading noise and discrete thickness noise. If the results achieved with both methodologies are compared, then it is possible to infer the specific contribution of every noise term (that is, thickness and loading) in the overall sound spectrum that the blower is radiating to the far field.

These formulations for both domains have been implemented using a $\mathrm{C}++$ algorithm. Pressure values and velocity components are extracted from the CFD modelling over the emission surfaces. This requires a statistic-stationary solution of the flow field in the modelling. Because this methodology is based on the resolution of the free-field FFWH equation, the acoustic model cannot consider those particular dispersion effects originated by any obstacle in the propagation region. At this point, the attenuation induced by the fan casing or the reverberations associated to the ground effect have been not introduced in the modelling. Future research will be oriented towards the inclusion of these type of mechanisms.

Since the acoustic sources are mainly concentrated over the blade surfaces, the modelled control surfaces have been defines coincident with the blades. Hence, the pressure distributions and the kinematics over the rotating blades are stored (for every cell) during the CFD computations comprising a complete rotor revolution.

When executing the computations in the temporal domain, the acoustic sources are evaluated in the retarded time through equation (10). The equation introduces a time lag between the noise emission instant and the moment of perception of the noise front wave at the observer's position in the far-field region. Therefore, to calculate the 360-deg. sound field at a fixed position, it is necessary to discretize all the possible observer's positions and determine the sound pressure level for every point. Following, the Fourier transform of these temporal signals is introduced to facilitate the comparison with the frequential methodology and the experimental measurements.
On the other hand, in the frequency domain, bipolar acoustic sources correspond to the harmonics of the blade loadings, which are obtained by means of the Fourier transform of the CFD temporal series. In this case, it is not necessary to resolve the equations at retarded time, with the consequent saving of computational cost.

Two nested summatories over all the harmonics of the blade loading (index $w$ ) and for all the harmonics of the blade passing frequency (index $m$ ) are established in equation (11). For a fixed value of $m$, it is possible to obtain the sound pressure level corresponding to the blade passing frequency $m \boldsymbol{B} \Omega$. Then, the contribution of every BPF harmonic to the total sound spectrum can be determined. Moreover, due to the characteristics of the Bessel functions, which are attenuated with the increasing order, only a reduced number of blade loading harmonics is really contributing to the sound level for a particular frequency component. As a practical consequence, the overall summatory converges very fast. The total sound spectrum will be the sum of all the contributions coming from every blades passing frequency obtained for all the values of the index $m$ (until final convergence is achieved).

The numerical results for the region of acoustic propagation have been obtained for three horizontal semi-planes situated at the blower axis height. Those semi-planes were placed inside the observation far field, with a radial distance respect to the blower rotor of $5 \mathrm{~m}, 7 \mathrm{~m}$ and $9 \mathrm{~m}$ for each one. Figure 2 showed a scheme with the location of all the planes.

The observer's locations, which correspond to the positions where the analysis of the noise radiated from the fan is going to be realized, have been placed then on those semi-planes, separated with a regular interval of $5^{\circ}$. The $-90^{\circ}$ angular position corresponds to an observation point located over the blower axis, stator upstream; meanwhile the $90^{\circ}$ angular position corresponds to an observer's point placed once again on the blower axis but now rotor downstream.

In the forthcoming noise analysis, the results obtained for the total SPL are shown, determined according to the next expression:

$$
S P L_{\text {total }}=10 \log \left(10^{S P L_{B P F} / 10}+\ldots+10^{S P L_{h B P F} / 10}\right)
$$

\section{RESULTS AND COMPARISON}

\section{Thickness noise and loading noise}

Following, the specific contribution of every type of acoustic source to the discrete noise radiated by the axial blower is analyzed. Figure 6 shows the results obtained at $\mathrm{R}=5 \mathrm{~m}$ for the SPL. The two different axial gaps are included in the figure. At the abscissa, the angular position of the observer is represented in degrees; in the vertical axis, the SPL is scaled in $\mathrm{dB}$. Discontinuous curves are showing results for the loading noise; meanwhile continuous ones correspond to the loading and thickness noise. Experimental results are also introduced. The difference between 
discontinuous and continuous curves is due to the effect of the thickness noise on the overall noise spectrum of the blower. For all the cases shown in Figure 6, the SPL values obtained using the integral formulation in the temporal domain ( $\mathrm{LN}+\mathrm{TN}$ curve) are higher than those obtained with the formulation in the frequency domain (LN curve). Differences are between 10 and $20 \mathrm{~dB}$ for the location situated perpendicular to the blower axis. This aspect shows that the contribution of the thickness noise is considerable, and for the particular case of this lowspeed axial fan, its influence cannot be neglected. Probably, these high values are a consequence of the great solidity of the rotor blades, so the displacement of fluid is an important noise source.

On the other hand, considering both lower and upper axial gaps, the results obtained for the loading and thickness noise are more similar with the experimental results than those obtained only for the loading noise, not only over the SPL magnitude itself but also for the spatial distribution of the SPL at plane $\mathrm{R}=5 \mathrm{~m}$. This fact is in consonance with the idea expressed before, where the thickness noise contribution was found to be relevant in the sound spectrum radiated into the far field.

All the differences observed for the SPL in the plots of Figure 6 between the numerical and the experimental results have several causes. Firstly, the real configuration of the axial blower tested in the lab is surrounded by an external shroud, which supports different parts of the fan structure, and obviously modifies the noise patterns radiated from the angular positions $-50^{\circ}$ to $50^{\circ}$, approximately. Besides, the experimental results include the noise radiated from the electric motor that drives the whole device. Secondly, the CFD modelling is not considering those noise sources related to the tip leakage flow. Therefore, tip vortex effects are not considered over the rotor blades as a new source of noise [14]. Finally, the existence of broadband noise for the experimental results must be considered (a contribution that has not been treated in the numerical modelling of the propagation region, due to the major interest placed on the discrete frequencies).

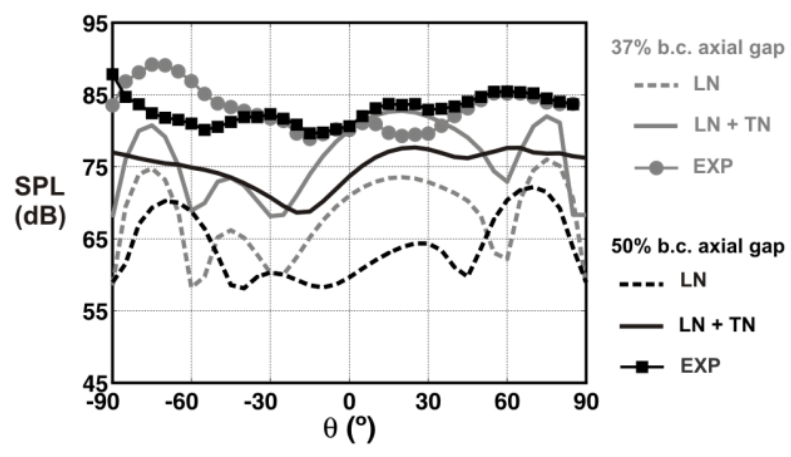

Figure 6. Thickness noise and loading noise.

With the formulation in the frequency domain ( $\mathrm{LN}$ curve), it can be said that less noise is propagated into the far field when the axial gap between the rows is larger. Because the forces exerted over the blades, as responsible of the loading noise, are lower for this configuration [13], it is expected that the generated loading noise is lower too. Now, for the temporal domain ( $\mathrm{LN}+\mathrm{TN}$ curve), this trend is not so evident, and even higher values for the SPL over the blower axis are obtained for the upper axial gap than for the lower. Besides, significant discrepancies appear over the circumferential distribution of the SPL along the R $=5 \mathrm{~m}$ plane, which must be assigned to the contribution of the thickness noise. These differences are increased when the separation between the rows is also increased. Experimentally, except for the zone affected by the shroud of the fan, it seems that less noise is radiated into the far field when the $50 \%$ b.c. axial gap is fixed in the test rig.

\section{SPL variation with the distance from the low-speed axial fan}

Figure 7 shows the variation of the sound level radiated to the far field as a function of the distance from the blower. The plots in Figure 7 show the circumferential distribution of the SPL at $\mathrm{R}=5,7$ and $9 \mathrm{~m}$ planes for both separations between the rows: $37 \%$ b.c. axial gap in the upper part of the figure and 50\% b.c. axial gap in the lower part. The first column shows the results corresponding to the loading noise; while the second one shows the contribution of both loading and thickness noise. A comparison with the experimental results is also provided in the third column.

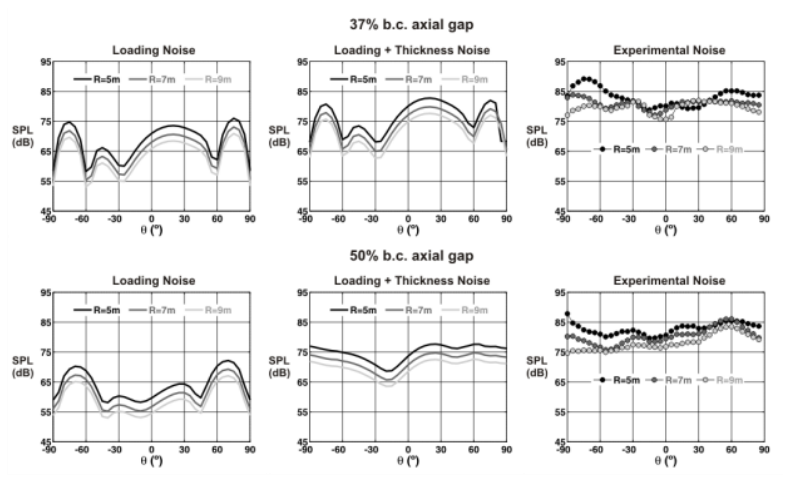

Figure 7. SPL variation with the distance from the axial blower.

As it can be observed, in all the cases the circumferential distributions of the SPL are similar for the three semi-planes. The difference between them is the reduction in the SPL magnitude as the distance increases from the low-speed axial fan. The differences between one plane and the next are estimated as 2.5 $\mathrm{dB}$, approximately. This behaviour agrees with the formulation of the aeroacoustic analogy, which predicts that the acoustic waves radiated to the far field are propagating like spherical waves.

For the SPL values measured from the experimental campaigns, an attenuation of the SPL magnitude with 
the distance can be also appreciated, except for the radiation zone affected by the presence of the fan shroud. In the case of the $50 \%$ b.c. axial gap, the attenuation of the experimental SPL is more evident than for the $37 \%$ b.c. axial gap.

\section{SPL spatial distribution}

Figure 8 shows the spatial distribution, for both circumferential and radial directions, of the SPL at the horizontal semi-plane that is placed at the axis height of the fan. The upper row shows the results corresponding to the loading and thickness noise; meanwhile the lower row summarises the experimental results. Besides, the plots in the left column correspond to the $37 \%$ b.c. axial gap configuration, and the plots in the right column to the $50 \%$ b.c. axial gap case.
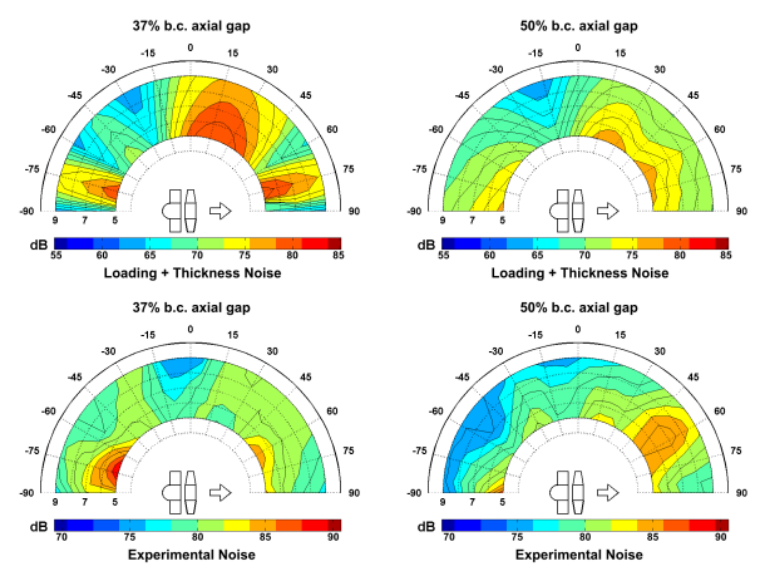

Figure 8. SPL spatial distributions.

In the case of the higher axial gap, a good agreement is found in the spatial distribution of the SPL level between experimental and numerical results. Though the levels are slightly higher in the experiments, due to the contribution of those additional sources not included in the modelling, the map reveals that the numerical methodology is capable to predict the higher propagation in the rear zones of the fan. In fact, the global distributions are quite similar between numerical and experimental results. However, when the gap is reduced to a $37 \%$ of the blade chord, there are major discrepancies in the distributions, especially at $20^{\circ}$ for the numeric. The prevalence of the loading noise over the thickness noise is probably responsible for this overestimation in the lower gap case. Anyway, it is noticeable that the experimental increase of the emission of noise at a circumferential position of $-75^{\circ}$ is well-predicted by the numerical code. The explanation for this feature becomes from a notable increase of the stator-rotor interaction, which is further traduced to a major unsteadiness on the blade forces, thus manifested upstream with important noise propagation. A symmetrical counterpart to this effect is also estimated in the numerical computations (at $75^{\circ}$ ).

Concerning the contribution of tonal noise, the sound pressure level calculated only with the BPF component is shown in figure 9 for the upper axial gap.
This polar representation is symmetric respect to the axis of the axial fan (the horizontal axis in the plots). The numerical results at 5, 7 and 9 meters from the fan location have been represented to track the attenuation of the noise level as before. The loading noise, represented in the left picture, reveals a clear dipole structure, with maximum values perpendicular to the fan axis. Though notable, just higher levels of roughly $55 \mathrm{~dB}$ are observed for the loading noise. As previously discussed, this contribution is especially sensitive to the axial gap. Hence, in the case of the reduced gap (not shown here) maximum values at $0^{\circ}$ reach up to $65 \mathrm{~dB}$. When the contribution of the thickness noise is added (central plot), the dipole structure is completely masked, and the propagation of the aerodynamic noise due to the displacement of the fluid by the blades becomes predominant. Close to the fan axis, the SPL is increased and the overall values reach up to nearly $70 \mathrm{~dB}$. The directivity pattern is lost and the features of the SPL turn to be omnidirectional. Finally, when compared to the experimental results, it is clear that higher levels are always observed when measuring (about $80 \mathrm{~dB}$ at 5 meters from the source) due to the influence of additional noise sources. However, the omnidirectional pattern of the experimental results at BPF is a clear indicator of the negligible influence of dipolar sources in the directivity patterns of the aerodynamic noise generated by this type of axial flow fans.

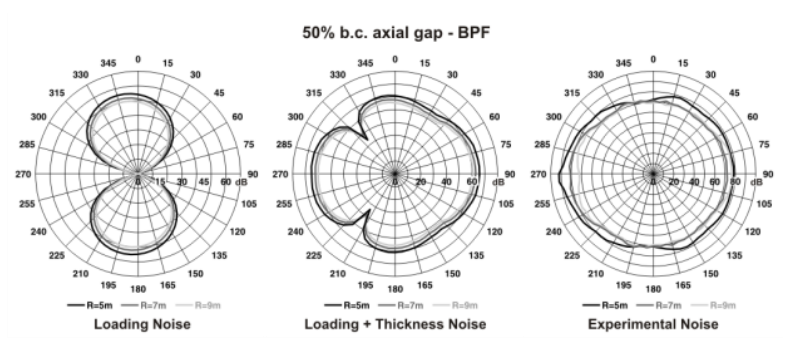

Figure 9. Spatial distribution of the SPL corresponding to the BPF component.

\section{Noise Spectra}

The representation of noise spectra is of particular interest when analyzing the contribution of tonal frequencies at BPF and its harmonics in the axial blower. Hence, in figure 11 , the noise spectrum obtained for the $50 \%$ b.c. axial gap configuration with the numerical model has been compared to the experiments. The chart corresponds to an angular position of the observer at $45^{\circ}$ over the $R=5,7$ and 9 $\mathrm{m}$ planes. Both numerical and experimental spectra are clearly dominated by the noise peaks at the blade passing frequency and its harmonics. Moreover, in the experimental spectrum, the baseline level is maintained all over the frequency range of the figure, indicating also the relevance of the broadband noise. Conversely, the numerical baseline is drastically reduced from 8 $\mathrm{kHz}$ on, revealing the lack of numerical accuracy in modelling the smallest scales of the turbulence structure. Therefore, much of the underestimation of 
SPL levels in the numerical predictions is due to the use of a coarse mesh, unable to capture high-frequency fluctuations. An additional representative characteristic is the prevalence of the tonal peak of the first harmonic at $720 \mathrm{~Hz}$, even more intense than the fundamental frequency, and perfectly followed by the numerical modelling.
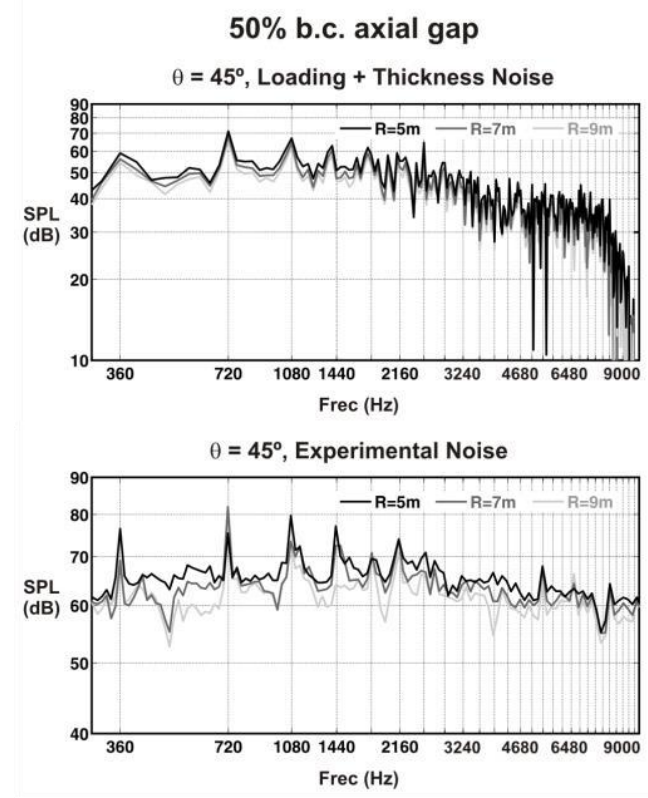

Figure 10. Noise spectrua at $R=5,7$ and $9 \mathrm{~m}$ planes.
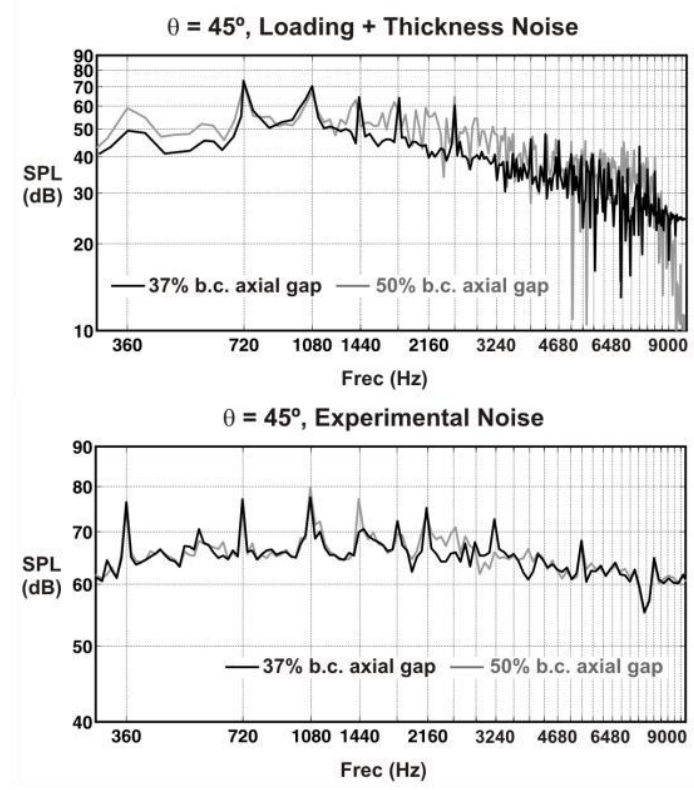

\section{Figure 11. Comparison of the noise spectra for both} gaps configurations at plane $R=5 \mathrm{~m}$.

Finally, figure 11 compares both numerical and experimental spectra 5 meters away from the source at a $45^{\circ}$ angular position, for the two axial gaps tested in the present investigation. Practically, no differences are observed in the experimental spectrum. Besides, the numerical spectra are similar, though the weight of the tonal noise is predominant with respect to the baseline levels for the $37 \%$ b.c. axial gap case.

\section{CONCLUSIONS}

A numerical methodology to predict the far-field aeroacoustic behaviour of a low-speed axial fan with inlet guide vanes has been developed. The methodology was applied to two different interrow spacings of the fan.

The numerical methodology was based on a hybrid formulation based in the FFWH aeroacoustics analogy, which segregates the problem of the noise source determination in the source region from the problem of the noise propagation into the far field.

The numerical characterization of the source region has been carried out with the implementation of a 3D CFD modelling of the complete axial fan, closing the turbulence modelling with a WMLES scheme.

The numerical characterization of the propagation region has been completed through an integral formulation for both frequency (loading noise) and temporal domains (loading and thickness noise) of the FFWH wave equation. For that purpose, a $\mathrm{C}++$ code has been implemented using an algorithm that takes as input data the acoustic sources that were identified in the CFD modelling at the source region. Finally, the numerical methodology has been compared with experimental results.

Analyzing the SPL results obtained in the far field, it can be concluded that the thickness noise has an significant contribution in the radiated sound spectrum, at least for this particular axial fan. Despite the lowvelocity at the tip of the rotor blades, the results confirm that their solidity cannot be neglected: blade rotation is displacing a considerable volume of fluid, generating a monopolar noise source that cannot be ignored. Besides, the contribution of the thickness noise becomes more relevant when the axial gap is increased and the stator-rotor interaction falls. This is clearly a consequence of the reduction in the force unsteadiness of the blades for lower axial gaps, manifested as a minor discrete loading noise.

Basically, the comparison between the numerical and the experimental results has shown a reasonable overall coherency. However, notable differences, especially in the noise levels rather than in the spatial distributions, arose due to the existence of broadband noise in the experimental results and the influence of the fan casing, not considered in the modelling. Future work will deal with the inclusion of these contributions of broadband noise.

A numerical tool has been advanced to be used in the design process of the machine in order to predict the aeroacoustic behaviour of a low-speed axial fan. Global trends can be identified to propose corrective actions concerning noise reductions even in the early stages of new fan developments.

\section{NOMENCLATURE}

b.c. Blade Chord 


\begin{tabular}{|c|c|}
\hline $\mathrm{BC}$ & Boundary Conditions \\
\hline BPF & Blade Passing Frequency \\
\hline CAA & Computational Aero Acoustics \\
\hline CFD & Computational Fluid Dynamics \\
\hline DNS & Direct Numerical Simulation \\
\hline FFWH & Ffowcs Williams and Hawkings \\
\hline IGVs & Inlet Guide Vanes \\
\hline LES & Large Eddy Simulation \\
\hline RANS & Reynolds Average Navier-Stokes \\
\hline RSM & Reynolds Stress Model \\
\hline SPL & Sound Pressure Level \\
\hline URANS & Unsteady Reynolds Average Navier-Stokes \\
\hline WMLES & Wall Modeled Large Eddy Simulation \\
\hline$B$ & Number of blades \\
\hline$c_{0}$ & Sound speed $[\mathrm{m} / \mathrm{s}]$ \\
\hline$C_{S}$ & Smagorinsky-Lilly model constant \\
\hline$f$ & Control surface \\
\hline$\vec{F}$ & Flow vector \\
\hline$F_{i}$ & Loading noise source term \\
\hline$F_{w V}$ & Blade loading harmonics $[\mathrm{N}]$ \\
\hline$g$ & Retarded time equation \\
\hline$\vec{H}$ & Source terms vector \\
\hline$H$ & Heaviside function \\
\hline$J$ & Bessel function \\
\hline$l_{i}$ & Blade loading force components $[\mathrm{N}]$ \\
\hline$M$ & Mach number \\
\hline$n_{i}$ & Normal components \\
\hline$p$ & Static pressure $[\mathrm{Pa}]$ \\
\hline$p^{\prime}$ & Acoustic pressure fluctuation $[\mathrm{Pa}]$ \\
\hline$p_{L}^{\prime}$ & Loading noise pressure $[\mathrm{Pa}]$ \\
\hline$p_{T}^{\prime}$ & Thickness noise pressure $[\mathrm{Pa}]$ \\
\hline$q$ & Thickness noise source term \\
\hline$v_{i}$ & Flow velocity components $[\mathrm{m} / \mathrm{s}]$ \\
\hline$V_{i}$ & Surface velocity components $[\mathrm{m} / \mathrm{s}]$ \\
\hline$\vec{r}$ & Vector position $[\mathrm{m}]$ \\
\hline$R$ & Observer radial coordinate $[\mathrm{m}]$ \\
\hline$R_{H}$ & Hub radius $[\mathrm{m}]$ \\
\hline$R_{0}$ & Spanwise coordinate $[\mathrm{m}]$ \\
\hline$R_{T}$ & Tip radius $[\mathrm{m}]$ \\
\hline $\operatorname{Re}_{d h}$ & Chord Reynolds number \\
\hline$S$ & Control surface area $\left[\mathrm{m}^{2}\right]$ \\
\hline$S_{M i}$ & Momentum source terms \\
\hline$t$ & Observer time $[\mathrm{s}]$ \\
\hline$T_{i j}$ & Lighthill tensor \\
\hline$V$ & Number of vanes \\
\hline$\vec{W}$ & Conservation variables vector \\
\hline$\vec{x}$ & Observer position $[\mathrm{m}]$ \\
\hline
\end{tabular}

\section{Greek letters}

$\delta \quad$ Dirac delta function

$\gamma \quad$ Inclination angle $\left[{ }^{\circ}\right]$

$\varphi \quad$ Observer angular coordinate $\left[{ }^{\circ}\right]$

$\begin{array}{ll}\varphi_{0} & \text { Chordwise coordinate }\left[^{\circ}\right] \\ \Omega & \text { Rotational velocity }[\mathrm{rad} / \mathrm{s}] \\ \theta & \text { Observer angular position }\left[^{\circ}\right] \\ \rho & \text { Flow density }\left[\mathrm{kg} / \mathrm{m}^{3}\right] \\ \rho_{0} & \text { Unperturbed flow density }\left[\mathrm{kg} / \mathrm{m}^{3}\right] \\ \sigma_{i j} & \text { Fluid stress tensor } \\ \tau & \text { Source time }[\mathrm{s}] \\ \tau_{i j} & \text { Viscous stress tensor }\end{array}$

\section{Subscripts}

$i=1,2,3$ Components

$j=1,2,3$ Components

$m \quad$ Blade passing frequency harmonics

$n \quad$ Normal direction

$r \quad$ Radial direction

$w \quad$ Loading harmonics

\section{ACKNOWLEDGEMENTS}

This work was supported by the Research Project "Characterization of the aerodynamic noise generation by rotor-stator interaction in axial flow fans", ref. DPI2006-15270, MEC.

\section{REFERENCES}

[1] Cumpsty N A 1977 A critical review of turbomachinery noise ASME Journal of Fluids Engineering 99 278-293

[2] Neise W 1976 Noise reduction in centrifugal fans: a literature survey Journal of Sound and Vibration 45 375-403

[3] Quinlan D A, Bent P H 1998 High frequency noise generation in small axial flow fans Journal of Sound and Vibration 218(2) 177-204

[4] Gérard A, Berry A, Masson P 2005 Control of tonal noise from subsonic axial fan. Part 1: reconstruction of aeroacoustic sources from farfield sound pressure Journal of Sound and Vibration 288 1049-1075

[5] Schöeder W, Ewert R 2004 Computational Aeroacoustics using the hybrid approach Von Karman Institute for Fluid Dynamics, Lecture Series 200405

[6] Djambazov G, Lai C H, Pericleous K A 1997 Domain decomposition methods for some aerodynamic noise problems Proceedings of the 3rd AIAA/CEAS Aeroacoustics Conference 191198

[7] Lighthill M J 1952 On sound generated aerodynamically. Part I: general theory Proceedings of the Royal Society A211 564-587

[8] Lighthill M J 1954 On sound generated aerodynamically. Part II: turbulence as a source of sound Proceedings of the Royal Society A222

[9] Ffowcs Williams J E, Hawkings D L 1969 Sound generation by turbulence and surfaces in arbitrary motion Phil. Trans. Royal Soc. A264 321-342 
[10] Neise W 1992 Review of fan noise generation mechanisms and control methods Proceedings Fan Noise Symposium Senlis 45-56

[11] Farassat F 1974 The acoustic far-field of rigid bodies in arbitrary motion Journal of Sound and Vibration 32(3) 387-405

[12]Blake W K 1986 Mechanics of flow-induced sound and vibration Academic Press, Inc.

[13] Fernández Oro J M, Argüelles K M, Santolaria C 2005 Unsteady rotor-stator interaction in an axial flow blower. Part I: numerical and experimental flow field characterization Proceedings of the 2005 ASME Fluids Engineering Division Summer Conference 1348-1357

[14] Wang M, Freund J B, Lele S K 2006 Computational prediction of flow generated sound Annual Review of Fluid Mechanics 38 483-512

[15]Fernández Oro J M, Argüelles Díaz K M, Santolaria C, Blanco Marigorta E 2007 Unsteady flow and wake transport in a low-speed axial fan with inlet guide vanes ASME Journal of Fluids Engineering 129 1015-1029

[16] Rossing T D (Ed) 2007 Springer Handbook of Acoustics Springer Science+Business Media, LLC New York ISBN: 978-0-387-30446-5

[17] Davidson L, Dahlström S 2004 Hybrid LESRANS: an approach to make LES applicable at high Reynolds numbers Proceedings of CHT-04 ICHMT International Symposium on Advances in Computational Heat Transfer

[18] Piomelli U 2005 Large eddy simulation and related techniques: theory and applications Von Karman Institute for Fluid Dynamics, Lecture Series 2005-06

[19] Argüelles Díaz K M, Fernández Oro J M, Santolaria C, Ballesteros-Tajadura R 2006 Tonal noise generation in an inlet vaned axial blower at several axial gaps Proceedings of the ASME Turbo Expo 2006 Barcelona 
This document is a pre-print version of the scientific paper published by SAGE Publishing. It has been released by the authors to fulfill all the publisher requirements established for Article Sharing:

https://uk.sagepub.com/en-gb/eur/journal-author-archiving-policies-and-re-use

\section{(c) 1 (i) (9)}

(C) 2019. This manuscript version is made available under the Creative Commons Attribution-NonCommercial-NoDerivatives 4.0 International License (CC-BY-NC-ND 4.0 license) http://creativecommons.org/licenses/by-nc-nd/4.0/ 